\title{
Emergency Medicine Physicians Performed Ultrasound for Pediatric Intussusceptions
}

\author{
Yi-Jung Chang, Shao-Hsuan Hsia, Hsun-Chin Chao
}

Background: Intussusception is the common acute abdomen in children with difficult clinical diagnosis. The routine ultrasound has recently been proposed as the initial diagnostic modality with high accuracy, but is not available for $24 \mathrm{~h}$ by gastroenterologists. We aimed to evaluate the validation of bedside ultrasound for intussusceptions performed by pediatric emergency physicians with ultrasound training during the night or holiday.

Methods: A retrospective study was conducted in children with suspected intussusceptions when routine ultrasounds by gastroenterologists were not available over the period from July 2004 to July 2008. Patients were divided into two groups: those diagnosed by emergency physicians with ultrasound training and without training. The clinical characteristics and course for all patients were reviewed and compared for seeking the difference.

Results: $\quad$ A total of 186 children were included. One hundred and thirteen $(61 \%)$ children were diagnosed by pediatric emergency physician with ultrasound training. The clinical symptoms were not statistically different between the two groups. The diagnostic sensitivity of the ultrasound training group was significantly higher $(90 \%$ vs. $79 \%, p=0.034)$. Children of the training group also had significantly shorter hospital stay duration at emergency departments before reduction $(2.41 \pm 2.01$ vs. $4.58 \pm 4.80 \mathrm{~h}, p=0.002)$.

Conclusion: Bedside ultrasound performed by pediatric emergency physicians with ultrasound training is a sensitive test for detecting intussusceptions. Knowledge and use of bedside ultrasound can aid the emergency physician in the diagnosis of pediatric intussusceptions with less delay in treatment. (Biomed J 2013;36:175-178)

Key words: child, intussusceptions, ultrasound

$\mathrm{I}_{\mathrm{in}}^{\mathrm{n}}$ ntussusception is the most common abdominal emergency in infants and young children and can potentially be fatal if not diagnosed and treated promptly. ${ }^{[1,2]}$ Ultrasound (US) has been used as the primary diagnostic procedure and has resulted in a reduction in the number of enemas for patients with intussusceptions. ${ }^{[3,4]}$ A negative sonographic diagnosis of intussusceptions avoids an unnecessary diagnostic air or

\begin{abstract}
At a Glance Commentary
Scientific background of the subject

Intussusception is the common abdominal emergency in children with difficult clinical diagnosis. The routine ultrasound is not available for $24 \mathrm{~h}$ by gastroenterologists. The aim of the study is to evaluate the validation of bedside ultrasound for intussusecptions performed by pediatric emergency physicians during the night or holiday.
\end{abstract}

\section{What this study adds to the field}

According to our study, bedside ultrasound performed by pediatric emergency physicians with ultrasound training is a sensitive test for detecting intussusceptions with less delay in treatment.

From the Division of Pediatric General Medicine, Department of Pediatrics, Chang Gung Memorial Hospital at Linkou, Chang Gung University College of Medicine, Taoyuan, Taiwan

Received: Feb. 23, 2012; Acceptance: Aug. 7, 2012

Correspondence to: Dr. Hsun-Chin Chao, Department of Pediatrics, Chang Gung Memorial Hospital at Linkou. 5, Fusing St., Gueishan, Taoyuan 333, Taiwan (R.O.C.). Tel: 886-3-3281200; ext. 8969; Fax: 886-3-3288957; E-mail: r64321@ yahoo.com.tw

DOI: $10.4103 / 2319-4170.112739$

barium enema. Because formal radiologic US is not available around the clock at some institutions, ${ }^{[5,6]}$ bedside US by pediatric emergency physicians may be a way to expedite and improve care in these situations. Emergency bedside ultrasound (EMBU) has been used in emergency departments for over two decades. ${ }^{[7,8]}$ One recent study found that $39 \%$ of pediatric emergency fellowship directors reported 
routine use of US by faculty. ${ }^{[9]}$ To our knowledge, no study has determined the usefulness of EMBU for intussusceptions when used by pediatric emergency medicine physicians. The aim of this study was to assess the validations of EMBU in the diagnosis of pediatric intussusceptions at the emergency department. Understanding the magnitude of this discrepancy may help to determine the best allocation of resources for pediatric intussusceptions' evaluation.

\section{METHODS}

A retrospective case series was conducted at the Chang Gung Children's Hospital over an 18-month period from July 2004 to June 2008. The institutional review board at our hospital approved this study.

The EMBU was performed by the pediatric emergency attending physicians who had completed the fundamental gastrointestinal US training course provided by the Pediatric Critical Care and Emergency Medicine Department of the Chang Gung Memorial Hospital (a 1-month training course in the use of US for the evaluation of the acute abdomen). Five pediatric emergency physicians in training $\left(1^{\mathrm{st}}-3^{\text {rd }}\right.$ year pediatric emergency attending physicians) were involved in this study; all of them had received standardized training in abdominal US that was supervised by gastroenterologists. Training included an average of over 100 supervised investigations of the abdomen. Patients were eligible for inclusion in the study if they were less than 18 years of age with clinically suspected intussusceptions (ICD-9 5600) at emergency departments during nights and weekends (when gastroenterologists were not available). Patients referring from the outpatient department or from other hospitals with US or lower gastrointestinal series reports were excluded from the final data analysis. The final diagnosis of intussusceptions was confirmed by air reduction. Patients were divided into two groups on the basis of being diagnosed by physicians with or without EMBU training. Patients were assigned to group I if they were diagnosed by pediatric attending physicians with EMBU training or to group II if they were diagnosed by those without EMBU training. The clinical course and outcome including clinical records and radiological reports were reviewed for each patient encounter. All data were compared between the two groups. Statistical analysis of the data was performed using the SPSS 17.0 software package. We applied the Fisher's exact test and the Mann-Whitney test to analyze the categorical and continuous variables as appropriate. A $p<0.05$ was considered significant.

\section{RESULTS}

During the study, data on a total of 520 children with a suspicion of intussusceptions were collected. Three hundred and thirty-seven (65\%) children visited the emergency department when experienced gastroenterologists were not available. One hundred and fifty-one patients were excluded for referring from other hospital and 186 patients were enrolled into this study. Of these 186 patients, 113 were directly managed by pediatricians with US training (group I) and 73 were managed by pediatricians without US training (group II). The median age of the 186 patients was 1.75 years and most of the patients were male $(60 \%)$. No statistically significant differences were found in age or gender between the two study groups.

The clinical symptoms between those two groups showed no statistically significant difference [Table 1]. Overall, 177 children had confirmed intussusceptions via air reduction. Of the reduction-confirmed cases, $5(3 \%)$ had vomiting as the only presenting symptom and $13(7 \%)$ had diarrhea without currant jelly stool. For patients with a history of bloody stools, $88 \%$ of group I underwent EMBU and $16 \%$ of group II were ordered US investigations, a difference that was statistically significant. Overall, ultrasounds were performed more frequently in group I than in group II patients ( $90 \%$ vs. $23 \%, p<0.001)$. US by gastroenterologists yielded a positive predictive value of $93 \%$, which was similar to EMBU by pediatric emergency physicians that produced a positive predictive value of $97 \%$. The overall sensitivity and positive predictive value of US by gastroenterologists or pediatric emergency physicians were higher than those with clinical diagnosis of group II ( $100 \%$ vs. $61 \%, p<0.001$ and $97 \%$ vs. $87 \%, p=0.029$ ).

The difference in diagnostic results and management between groups I and II is shown in Table 2. The diagnostic sensitivity and positive predictive value for intussusceptions in group I were statistically significantly higher than those in group II (90\% vs. $79 \%, p=0.034$ and $97 \%$ vs. $88 \%, p=0.029$, respectively). All false-negative cases, including 11 of group I

Table 1: Clinical characteristics

\begin{tabular}{lccc}
\hline & Group I $n=113(\%)$ & Group II $n=73(\%)$ & $p$ \\
\hline Irritable & 42.5 & 39.7 & 0.710 \\
Vomiting & 39.8 & 53.4 & 0.069 \\
Bloody stool & 15.9 & 24.7 & 0.141 \\
Diarrhea & 11.5 & 15.1 & 0.479 \\
Fever & 10.6 & 16.4 & 0.248 \\
\hline
\end{tabular}

Table 2: Summary of results

\begin{tabular}{lccc}
\hline & Group I & Group II & $p$ \\
\hline False negative & 11 & 14 & \\
False positive & 3 & 7 & \\
True positive (sensitivity) & $99 / 110(90 \%)$ & $52 / 66(79 \%)$ & 0.034 \\
Positive predictive value & $99 / 102(97 \%)$ & $52 / 59(88 \%)$ & 0.029 \\
Duration before reduction (h)* & $2.41 \pm 2.01$ & $4.58 \pm 4.80$ & 0.002 \\
US by gastroenterologists & $0 \%$ & $23 \%$ & $<0.001$ \\
\hline
\end{tabular}

*Values are mean \pm SD 
and 14 of group II, were admitted with other diagnoses due to dehydration or intractable abdominal pain. Children of group I without the EMBU had several misdiagnoses. The most common misdiagnosis was acute gastritis (73\%). For $3(18 \%)$ of the children in group II, with US examination, the air reduction was prevented due to the negative sonographic reports resulting in a smooth clinical course. Three (3\%) children of group I with EMBU and $1(6 \%)$ child of group I with US had false-positive results of intussusceptions. The false-positive predictive value was not statistically different between the children evaluated using EMBU by pediatric emergency physicians or US by gastroenterologists ( $97 \%$ vs. $94 \%, p=0.465)$. Of the children with a false-positive diagnosis in group II, $70 \%$ had abdominal pain or irritable crying and 30\% had bloody stools. Children of group I also had significantly shorter mean emergency department stays (before air reduction) $(2.41 \pm 2.01$ vs. $4.58 \pm 4.80 \mathrm{~h}, p=0.001)$ and required fewer US examinations by gastroenterologists at emergency departments ( $0 \%$ vs. $23 \%, p<0.001)$ than those of group II. One child of group II had a computed tomography (CT) examination. The successful air reduction rates in groups I and II were $86 \%$ and $75 \%$, respectively. No mortality was noted during the course of the study.

\section{DISCUSSION}

The clinical diagnosis of intussusceptions can be very difficult, with up to $50 \%$ of patients having nonspecific presentations at emergency department. The painless intussusceptions, vomiting, or diarrhea without currant jelly stool caused diagnostic challenges for patients with acute gastroenteritis. Therefore, a large percentage of clinical misdiagnoses were made at our emergency department. Currently, there is no reliably predictive model based on clinical findings that can accurately identify all patients with intussusceptions. ${ }^{[10]}$ US has become the modality of choice to aid in the diagnosis of intussusceptions. In skilled hands, US has high sensitivity (98-100\%) and specificity (88-100\%) for the diagnosis of intussusceptions. ${ }^{[1,11]}$ In our series, the diagnostic sensitivity and positive predictive value with US for intussusceptions were higher than those from clinical findings. Although US is operator dependent, our study suggests a high sensitivity and positive predictive value, which are independent of the performer. This high sensitivity and positive predictive value may be due to the characteristic sonographic appearance of intussusceptions, which facilitates diagnosis and exclusion. ${ }^{[12,13]}$ In our hospital, over half of the children visited the emergency department during nights or weekends when experienced gastroenterologists were not available. Many institutions do not have US capabilities in-house $24 \mathrm{~h}$ a day. EMBU training should be considered for the potential benefits in the diagnosis of pediatric intussusceptions when formal US is not available.
The diagnostic sensitivity achieved by physicians with EMBU training is significantly higher than for those without training. Studies by Shanbhogue et al. and others also suggest a few months training of US is sufficient for a highly accurate investigation of clinically suspected intussusceptions. ${ }^{[14-16]}$ The clinical signs and symptoms of intussusceptions may overlap with other common conditions. ${ }^{[17]}$ The higher sensitivity achieved in group I may have been due to more US procedures being performed on the nonspecific clinical presentations. To our knowledge, there is no child with a missed diagnosis of intussusceptions when the evaluation was performed by EMBU. However, there were still some missed intussusceptions diagnosed by physicians with US training. The high index of clinical suspicion, with the aid of EMBU, may help physicians to evaluate the clinical and diagnostic challenge and prevent the misdiagnosis.

The classic triad of intermittent abdominal pain or irritable crying, a palpable mass, and red currant jelly stools is reported to have a positive predictive value of $93 \%$ for intussusceptions. ${ }^{[18]}$ Most of the false-positive patients in our study did not have the classic triad of clinical signs. Of our patients with bloody stools, majority in group II underwent reduction instead of waiting for US investigations at daytime. The diagnostic pitfalls in most intussusceptions and the care of possible complications or fatality with missing diagnosis of intussusceptions may contribute to the major cause of false-positive cases in the clinical diagnosis group. The false-positive cases with EMBU may be seen in conditions with a thickened bowel wall, such as enterocolitis. ${ }^{[19]}$ With the higher positive predictive value of EMBU, we were able to eliminate the need for enema examinations and the associated radiation. Enema is a more invasive diagnostic procedure that has the risk of perforation. ${ }^{[20]}$ Cancellation of the enemas also resulted in avoidance of the unpleasant experience, with all the attendant anxiety and concern, in young children. ${ }^{[21]}$

In our study, physicians, with the use of EMBU, had an early diagnosis with a decrease in the length of emergency department stay and a reduced charge for further imaging studies. Waiting for gastroenterologists or radiologist experienced in US diagnosis is costly in terms of time. The rapid emergency treatment of intussusceptions is essential in order to minimize complications such as bowel necrosis, perforation, and the need for resection of a segmental bowel. ${ }^{[22]} \mathrm{In}$ addition, emergency room physicians skilled in US diagnosis of intussusceptions may decrease the healthcare costs by using fewer imaging studies. ${ }^{[19,23]}$

Clearly, EMBU offers a quick, low-cost, and reliable method for detection of intussusceptions without exposure to radiation when experienced US technicians/operators are not available in our hospital. EMBU also has some potential 
advantages. It can be repeated at anytime if necessary. There is a high level of patient comfort and safety with US. ${ }^{[16]}$ Furthermore, there are no contraindications for a primary conservative approach with US in the stable child who is in good clinical condition. ${ }^{[24]}$

Our study was retrospective and based on a database by ICD code. Therefore, certain study limitations could occur. First, the true negative sample was limited in our data. Second, some confounding factors including the experience and subspecialty of pediatric emergency staff may influence the outcomes. A further prospective cohort study is mandatory to eliminate the conditions.

In conclusion, the use of EMBU as a primary investigation for children with suspected intussusceptions prevents unnecessary radiological procedures and hospital emergency department visits. We recommend caution and knowledge of the sonographic appearance of intussusceptions when using the EMBU by physicians with US training may help to diagnose pediatric intussusceptions at emergency departments when formal radiologic US is not available.

\section{REFERENCES}

1. Justice FA, de Campo M, Liem NT, Son TN, Ninh TP, Bines JE. Accuracy of ultrasonography for the diagnosis of intussusception in infants in Vietnam. Pediatr Radiol 2007;37:195-9.

2. Huang WS, Changchien CS, Lu SN. Adult intussusception: A 12-year experience, with emphasis on etiology and analysis of risk factors. Chang Gung Med J 2000;23:284-90.

3. Shanbhogue RL, Hussain SM, Meradji M, Robben SG, Vernooij JE, Molenaar JC. Ultrasonography is accurate enough for the diagnosis of intussusception. J Pediatr Surg 1994;29:324-7.

4. del-Pozo G, Albillos JC, Tejedor D. Intussusception: US findings with pathologic correlation - the crescent-in-doughnut sign. Radiology 1996;199:688-92.

5. Bines JE, Ivanoff B, Justice F, Mulholland K. Clinical case definition for the diagnosis of acute intussusception. J Pediatr Gastr Nutr 2004;39:511-8.

6. Burr A, Renaud EJ, Manno M, Makris J, Cooley E, DeRoss A, et al. Glowing in the dark: Time of day as a determinant of radiographic imaging in the evaluation of abdominal pain in children. J Pediatr Surg 2011;46:188-91.

7. Levy JA, Noble VE. Bedside ultrasound in pediatric emergency medicine. Pediatrics 2008;121:e1404-12.

8. Ramirez-Schrempp D, Dorfman DH, Tien I, Liteplo AS. Bedside ultrasound in pediatric emergency medicine fellowship programs in the United States: Little formal training. Pediatr Emerg Care 2008;24:664-7.

9. Chen L, Santucci A. Probing the availability of emergency ultrasound in pediatrics [abstract]. Pediatr Emerg Care 2006;22:688.

10. Klein EJ, Kapoor D, Shugerman RP. The diagnosis of intussusception. Clin Pediatr 2004;43:343-7.

11. del-Pozo G, Albillos JC, Tejedor D, Calero R, Rasero M, de-la-Calle U, et al. Intussusception in children: Current concepts in diagnosis and enema reduction. Radiographics 1999;19:299-319.

12. del-Pozo G, Albillos JC, Tejedor D, Calero R, Rasero M, de-la-Calle U, et al. Intussusception: US findings with pathologic correlation- the crescent-in-doughnut sign. Radiology 1996;199:688-92.

13. Bhisitkul DM, Listernick R, Shkolnik A, Donaldson JS, Henricks BD, Feinstein KA, et al. Clinical application of ultrasonography in the diagnosis of intussusception. J Pediatr 1992;121:182-6.

14. Daneman A, Navarro O. Intussception part 1: A review of diagnostic approaches. Pediatr Radiol 2003;33:79-85.

15. Verschelden P, Filiatrault D, Gare L, Grignon A, Perreault G, Boisvert $\mathrm{J}$, et al. Intussusception in children, reliability of US diagnosis-a prospective study. Radiology 1992;8:343-7.

16. Vasavada P. Ultrasound evaluation of acute abdominal emergencies in infants and children. Radiol Clin North Am 2004;42:445-6.

17. Kuppermann N, O'Dea T, Pinckney L, Hoecker C. Predictors of insussusception in young children. Arch Pediatr Adolesc Med 2000;154: 250-5.

18. D'Agostino J. Common abdominal emergencies in children. Emerg Med Clin North Am 2002;20:139-53.

19. Kairam N, Kaiafis C, Shih R. Diagnosis of pediatric intussusception by an emergency physician-performed bedside ultrasound: A case report. Pediatr Emerg Care 2009;25:177-80.

20. Applegate KE. Clinically suspected intussusception in children: Evidence-based review and self-assessment module. AM J Roentgenol 2005;185:s175-83.

21. Henrikson S, Blane CE, Koujok K, Strouse PJ, DiPietro MA, Goodsitt MM. The effect of screening sonography on the positive rate of enemas for intussusception. Pediatr Radiol 2003;33:190-3.

22. Lehnert T, Ina S, Till H, Rolle U. Intussusception in children-clinical presentation, diagnosis and management. Int J Colorectal Dis 2009; 24:1187-92.

23. Bucher BT, Hall BL, Warner BW, Keller MS. Intussusception in children: Cost-effectiveness of ultrasound vs diagnostic contrast enema. J Pediatr Surg 2011;46:1099-105.

24. Daneman A, Navarro O. Intussusception. Part 2: An update on the evolution of management. Pediatr Radiol 2004;34:97-108. 